\title{
Analog Modeling of Worm-Like Chain Molecules Using Macroscopic Beads-on-a-String
}

\section{Citation}

Tricard, Simon, Efraim Feinstein, Robert F. Shepherd, Meital Reches, Phillip W. Snyder, Dileni C. Bandarage, Mara Prentiss, and George M. Whitesides. 2012. Analog Modeling of Worm-Like Chain Molecules Using Macroscopic Beads-on-a-String. Physical Chemistry Chemical Physics 14, no. 25: 9041-9046.

\section{Published Version}

doi:10.1039/c2cp40593h

\section{Permanent link}

http://nrs.harvard.edu/urn-3:HUL.InstRepos:11920044

\section{Terms of Use}

This article was downloaded from Harvard University's DASH repository, and is made available under the terms and conditions applicable to Open Access Policy Articles, as set forth at http:// nrs.harvard.edu/urn-3:HUL.InstRepos:dash.current.terms-of-use\#OAP

\section{Share Your Story}

The Harvard community has made this article openly available.

Please share how this access benefits you. Submit a story.

\section{Accessibility}




\section{Analog Modeling of Worm-Like Chain Molecules Using Macroscopic Beads-on-a-String}

Simon Tricard, a,* Efraim Feinstein, ${ }^{\mathrm{b}}$ Robert F. Shepherd, ${ }^{\mathrm{a}}$ Meital Reches, ${ }^{\mathrm{a}}$ Phillip W. Snyder, ${ }^{\mathrm{a}}$ Dileni C. Bandarage, ${ }^{\mathrm{a}}$ Mara Prentiss, ${ }^{\mathrm{b}}$ and George M. Whitesides ${ }^{\mathrm{a}, \mathrm{c}, *}$

a Department of Chemistry and Chemical Biology, Harvard University, 12 Oxford Street, Cambridge, MA 02138, USA

${ }^{\mathrm{b}}$ Department of Physics, Harvard University, 17 Oxford Street, Cambridge, MA 02138, USA

${ }^{\mathrm{c}}$ Kavli Institute for Bionano Science \& Technology, Harvard University, 29 Oxford Street, Cambridge, MA 02138, USA

* Authors to whom correspondence should be addressed: stricard@gmwgroup.harvard.edu, gwhitesides@gmwgroup.harvard.edu 


\begin{abstract}
This paper describes an empirical model of polymer dynamics, based on the agitation of millimeter-sized polymeric beads. Although the interactions between the particles in the macroscopic model, and those between the monomers of molecular-scale polymers, are fundamentally different, both systems follow the Worm-Like Chain theory.
\end{abstract}




\section{Introduction}

Current computational simulations can not accurately quantify the very large number of interactions and conformations required to describe molecular phenomena (for example, polymer dynamics, solvation, crystal nucleation and growth, molecular recognition, etc.). Descriptions of the kinetics of dynamic phenomena are mostly unapproachable without drastic simplifications. Assumptions and approximations - some major - are required to make aspects of static and equilibrium problems tractable for theoretical modeling or simulation. Although we applaud the value of digital, computational models, ${ }^{1-4}$ we also believe that analog, physical methods have a role to play in understanding molecular (and supramolecular) phenomena, and we are exploring such models as a complement to theory and in silico simulation.

The models we are testing do not provide quantitative details of molecular properties; rather, they are intended to improve and test our intuition concerning the effect of mechanical agitation on the evolution of the conformation of multiparticle systems. As a first step to explore their dynamics, we have constructed an analog, physical model using several simplifications: (i) a relatively small numbers of macroscopic particles, (ii) a two-dimensional (2D) configuration, and (iii) agitation using mechanical stimulation (e.g., shaking). Our physical model gives an alternative to the analytical description and computational simulation of the dynamics of polymer behavior.

Interpretation of the behavior of multiparticle molecular systems often rests on important and not easily verified assumptions (e.g., the ergodic hypothesis) ${ }^{5}$ in addition, information essential to a complete interpretation of the behavior of individual constituents is not available, and is subsumed into observable collective properties. The development of simplified systems in which all the particles can be visualized and tracked, and in which the interactions and the nature 
of the agitation that drives the evolution of the system with time can be controlled, is broadly relevant to study complex molecular behaviors.

We propose macroscopic agitation (which we abbreviate "MacAg”) as a strategy for physical simulation of the behavior of microscopic systems. In this paper, we demonstrate that a MacAg simulation of a short-chain polymer reproduces Worm-Like Chain (WLC) behavior. ${ }^{6}$ We do not claim that this model mimics molecular interactions, because the origins of the interactions at the macroscopic and microscopic scales are fundamentally different. Instead, we have used MacAg to simulate a specific, focused question concerning the dynamics of short-chain molecules: that is, the temporal evolution of the end-to-end distances of short chains of beads threaded on a flexible string as they were agitated by shaking on a 2D surface. The data describing the end-to-end distances are compatible with predictions of the WLC theory: in particular, we observed good agreement between this theory and the relationship between oligomer length and persistence length for the macroscopic beads-on-a-string model.

We controlled the persistence length of the physical system by modifying the parameters of the chain (e.g., the composition and the shape of the beads, and the diameter of the thread) and the parameters of the agitation (e.g., the nature, the amplitude and the frequency of the shaking motion). We observed a correlation between the persistence length of the beaded string and the frequency of agitation. This dependence suggests that this type of mechanical agitation - which in this system is manifestly different from the agitation experienced by molecules in solution can be considered, in some sense, to be analogous to temperature.

Experimental models of "beads-on-a-string" simulate aspects of the physics of granular materials. Examples include the influence of topological constraints such as knots, the effect of confinement of the chain on a circular vibrating bed, and phase transitions, including glass transitions in polymers. ${ }^{7-10}$ The present paper models the dynamics of long-chain molecule 
conformation under agitation. Experiments involving macroscopic particles make it straightforward to control a number of the physical parameters that define these systems: for example, the nature and strength of the interactions (e.g., electrostatic or magnetic) between the particles, the energy transferred to the system (e.g., the amplitude and frequency of agitation), and anisotropy in the motion used to induce energy introduced into the system (e.g., by orbital or linear agitation). Here we provide a preliminary description of the capability of MacAg to follow one aspect of the behavior of microscopic polymers: compatibility with the WLC model. In this analog simulation, the only interactions between the particles of the chain are the physical contact and momentum transfer by collision, and by friction with the surface of the container driven by agitation of the experimental apparatus.

In addition, we employed Monte-Carlo simulations to rationalize the dynamics of the macroscopic experimental model. We found that a digitally simulated system, which behaves according to the limited set of rules that we propose as the driving forces in the MacAg simulations, also behaves in accordance with the WLC model.

\section{Background}

We initiated our work using MacAg in studies of the crystallization of Coulombic crystals. ${ }^{1-3}$ Beads that were electrostatically charged (by contact electrification) with opposite charge crystallized into regular and partially ordered arrays when agitated on a flat surface. ${ }^{2,3}$ These systems also showed complex phenomena that resembled phase separation. When these beads were connected on a string, they became a model for polymeric or oligomeric molecules. ${ }^{4}$ Examination of the conformations of these polymer models demonstrated (for appropriate designs of the sequences of beads) hairpin loops; this type of intrachain molecular recognition was an analogy for the folding in short-chain RNA in the molecular world. ${ }^{4}$ The driving force of 
the self-assembly was provided by an agitation system that transferred momentum to the particles through friction between the support surface and the particles, and by collisions of the particles with the wall of the container of the experimental apparatus. In these cases, the movement of the particles and their interactions were strongly influenced by electrostatic charging, and by the electrostatic interactions between charged particles.

The present study was based on experiments without electrically charging of the components; we focused on the simplest behavior of the polymer chain under mechanical agitation, with no influence from electrostatic interactions. Under the conditions of the experiments we described, the bead-on-a-string system was surprisingly well described by the WLC theory.

\section{Worm-Like Chain Model}

The WLC - also called the Porod-Kratky Chain model ${ }^{11}$ - describes a polymer or an oligomer as a string of contour length $L$ with a persistence length $L_{p}$. The contour length $L$ is defined as the length of the chain at its maximal extension. The parameter $L_{p}$ is described as "the scale over which the tangent-tangent correlation function decays along the chain”, and quantifies the stiffness of the chain. ${ }^{6,12}$ If $L_{p}$ is large compared to $L$, the chain is rigid; if $L_{p}$ is small compared to $L$, the chain is flexible. According to the WLC model, ${ }^{6}$ eq. 1 described the meansquare end-to-end distance $<R^{2}>$ as a function of $L$ and $L_{p}$.

$$
\left\langle R^{2}\right\rangle=2 \mathrm{~L}_{p} L\left(1-\frac{L_{p}}{L}\left(1-e^{-L / L_{p}}\right)\right)
$$

The WLC theory is a purely conformational description of a flexible long chain: it includes no specific interactions (attractive or repulsive), no size effects, and no time dependence. In this model, the polymer is considered as a one-dimensional (1D), inextensible and 
continuously flexible object; $L_{p}$ is the single parameter necessary to describe the stiffness of the chain. The calculation of $<R^{2}>$ comes from integration along the $1 \mathrm{D}$ curvilinear coordinate. Eq. 1 will thus always be an accurate description, whether we consider the chain in a two-dimensional (2D) or three-dimensional (3D) space. ${ }^{6}$ The WLC model is typically used to describe stiff polymers, as it does not take into account the self-avoidance constraint present in real systems. The WLC model is nonetheless the most used system to describe the dynamics of polymers, especially biopolymers like DNA and small unfolded proteins. ${ }^{13-15}$

The dependence of the persistence length of DNA on temperature is not obvious - in part because it is strongly influenced by interactions among ions, the charged backbone of DNA, and

the solvent - and this dependence is still an active area of study. ${ }^{16}$ More generally, the persistence length of a polymer is related to the temperature, $T$, according to eq. 2 , where $g$ is the bending constant of the polymer given in $\AA$.kcal.mol ${ }^{-1}$, and $R$ is the gas constant. ${ }^{17,18}$ A consequence of eq. 2 is that if the temperature has no effect on the bending rigidity of the system, $L_{p}$ will decrease with increasing temperature.

$$
L_{p}=\frac{g}{R T}
$$

\section{Experimental Design}

Our simple physical model can reproduce elements of polymer dynamics described by the WLC model. The macroscopic nature of the beads makes it possible to visualize in detail the motion of the chain with a specificity and clarity that is not possible with molecular species. This capability will help to understand the implications of theory for conformational behavior. The mean-square end-to-end distance is a key parameter in polymer statistical physics, and is the one we use to make a connection between the molecular and the macroscopic worlds. Molecular end- 
to-end distances can be measured experimentally by a variety of methods, either collectively in solution by Fluorescence Resonance Energy Transfer, or individually on single molecules using optical or magnetic tweezers, Atomic Force Microscope traps and single-molecule Fluorescence

Resonance Energy Transfer. ${ }^{14,15,19-23}$ The macroscopic experimental model makes it very easy to follow the end-to-end distance visually and thus to characterize the statistics of the chain.

The model, however, differs manifestly in its behavior from that of molecules because of: (i) the nature of the dominant forces at the macroscopic and microscopic scales, and the response of the chains to them; (ii) the experimental difficulty in the macroscopic model to generate purely random motion of the chains through mechanical agitation because the direction of transfer of momentum is the same on all points of the chain. The simplicity of the macroscopic system, and the obvious similarities and differences between the statistical description of the macroscopic chain and polymers in solution still makes this model a useful tool to study complex behaviors in macromolecules, such as the dynamics of homopolymers in solution.

\section{Physical Experiments}

The apparatus for agitation has been previously described (see Fig. S1 and materials and methods in supplementary information). ${ }^{4}$ Agitation occurred on a flat surface covered with paper and connected to an orbital shaker with variable frequency control. The roughness of paper assured that the beads rolled and did not slide. A pendulum attached under the platform, and excited by a linear motor, randomized the movement. We placed chains of beads of different natures and lengths on the surface (Fig. 1a). We threaded Nylon beads (6-mm in diameter; we use the term "beads" to describe both spheres and cylinders) on Nylon threads to generate the chains. 
Figure 1. a) Schematic representations of "beads-on-a-string” chains comprising either four spherical beads or three cylindrical beads. The beads at the termini are dyed in blue for visual contrast in image analysis. Each sphere or cylinder is separated from one another by three 3-mm balls (grey) and two crimp tubes that assure the spacing and flexibility of the chain (black). b) $R$ represents the end-to-end distance measured from the images. c) A graphical representation of the model used for Monte-Carlo simulation with four large mobile beads (green); each bead is separated from one another by three small mobile beads (green) and two fixed beads that constrain the movement of the mobile beads on the string (purple). d) Representative digital images captured under agitation of spheres-on-a-string of different lengths $L(4,7,11,14,17$, and 21 spheres per string corresponding to $L=5.8,11.6,19.4,25.2,31.0$ and $38.8 \mathrm{~cm})$. e) Representative images captured under agitation of cylinders-on-a-string of different lengths $L$ (3, $5,8,10,12$, and 15 cylinders per string corresponding to $L=5.4,10.7,18.4,23.9,29.2$, and 37.5 $\mathrm{cm})$. Scale bars: $2 \mathrm{~cm}$. 
a)

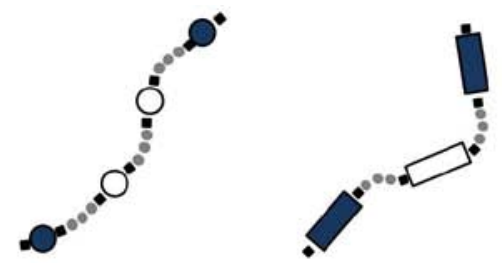

b)

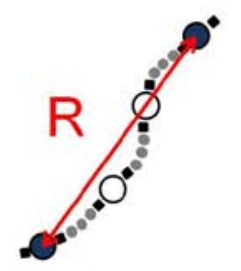

c)

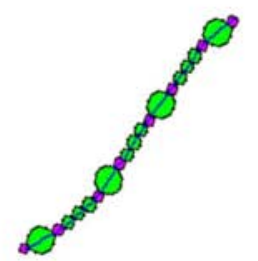

d)

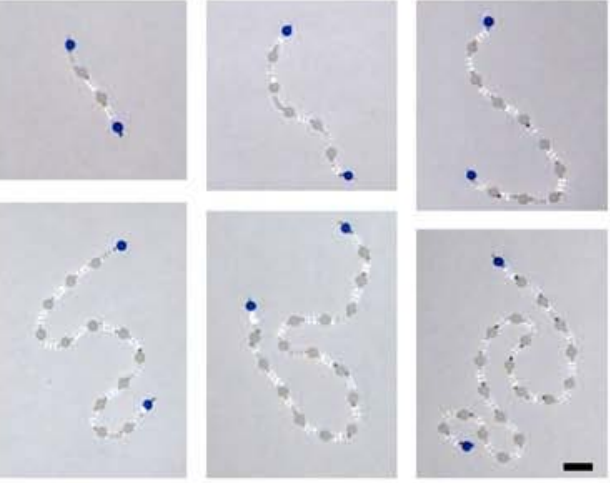

e)

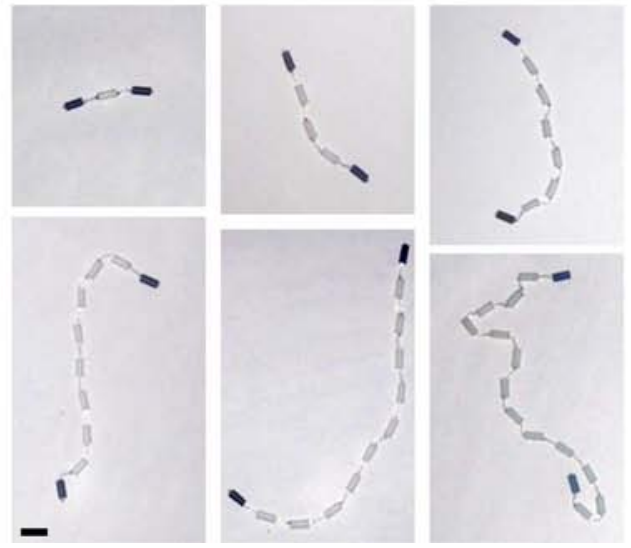


These large beads were separated by three small acrylic spheres (3-mm in diameter), which we inserted between two metal crimp tubes to maintain the flexibility of the chain. ${ }^{4}$ We used either sphere-shaped beads or cylinder-shaped beads to modify the rigidity of the chain. Increasing the diameter of the thread (from $75-\mu \mathrm{m}$ to 150 - $\mu \mathrm{m}$ in diameter) lowered the flexibility of the chain.

We agitated the beads-on-a-string at different frequencies, $f$, from 120 to 160 rounds per minute (rpm). ${ }^{24}$ We dyed one bead at each terminus of the string to make it easier to determine the end-to-end distance, which was measured from the middle of the two terminal beads (Fig. 1b). We agitated the chains for durations ranging from 100 to $200 \mathrm{~min}$, while capturing a single digital image at regular intervals (every 30 s). We coded an image analysis program in Matlab to determine the end-to-end distance on each frame. We calculated the mean-square end-to-end distance $<R^{2}>$ over a large number of snapshots (between 200 and 400) for different lengths of chains (Fig. 1d and 1e) and compared it to the analytical WLC model.

\section{Monte-Carlo Simulation}

We performed Monte-Carlo computer simulations using a model with digital parameters equivalent to those of our physical beads-on-a-string model. The string was a fixed length 1D object and, as in the mechanical model, we simulated three types of beads: large beads, small beads, and small fixed beads (Fig. 1c). We centered fixed beads at defined positions along the string and fixed the string straight inside each bead from its entry point to its exit point. At the entry and exit points, the string was allowed to bend at any angle between -90 degrees and 90 degrees. Bends greater than this range would cause the beads to overlap. Non-fixed beads could shift in one dimension into adjacent voids along the string. Beads were not allowed to overlap in space. 
We controlled the chain flexibility by controlling the ratio of the total length of the string to the length of the string inside the beads, which corresponded to the sum of the diameters of the beads. For example, a chain of flexibility 1.0 was unable to bend, whereas chains of flexibility 1.01, with $1 \%$ exposed string length, and a chain of flexibility 1.20 , with $20 \%$ of its length exposed, might both bend without the beads overlapping in space. We measured the fluctuation in mean-square end-to-end distance $<R^{2}>$ of chains with the same number of beads as the analog physical system and compared the results to the analytical WLC model. Lengths are given in arbitrary units (a.u.); one a.u. corresponds to the distance between two nearest beads in a totally extended conformation.

\section{Results and Discussion}

Behavior of Macroscopic Beads-on-a-String under Agitation.

We considered three different chains of beads: two chains of spherical beads (6 mm in diameter) threaded on different threads (75 or $150 \mu \mathrm{m}$ in diameter), and one chain of cylindrical beads threaded on a 75- $\mu$ m-diameter thread. Under agitation over a period of 100 to 200 min, the chains bent and translated across the surface. Fig. 2a shows the dependence of $\left\langle R^{2}\right\rangle$ on the contour length, $L$, calculated from the experimental data. We fit the data with the WLC model formula given in eq. 1 and found excellent agreement, $\left(r^{2}>0.99\right)$. This correlation showed an increase in $L_{p}$ while increasing the diameter of the thread $\left(L_{p}=3.6 \mathrm{~cm}\right.$ for a $75 \mu \mathrm{m}$-diameter thread and $L_{p}=7.8 \mathrm{~cm}$ for a $150 \mu \mathrm{m}$-diameter thread). Altering the geometry of the polymeric beads had a drastic effect on the persistence length of the chain. For the same diameter of thread, $L_{p}$ was $7.8 \mathrm{~cm}$ for spherical beads, and $12.2 \mathrm{~cm}$ for cylindrical beads. We are thus able to tune the persistence length of the system by adjusting either the diameter of the thread or the shape of the beads. 
Figure 2. a) Mean-square end-to-end distance as a function of the length of the chain for spheres on a $75 \mu \mathrm{m}$-diameter thread, for spheres on a $150 \mu \mathrm{m}$-diameter thread, for cylinders on a $75 \mu \mathrm{m}$ diameter thread, and their corresponding $L_{p}$ calculated using the Worm-Like Chain model. The frequency of agitation was $140 \mathrm{rpm}$. b) Mean-square end-to-end distance as a function of the length of the chain obtained by Monte-Carlo simulation for flexibilities equal to 1.01, 1.02, 1.05, 1.1, 1.2, and their corresponding $L_{p}$ calculated using the Worm-Like Chain model. See the text for the definition of flexibility. Error bars are 95\% confidence intervals.

a)

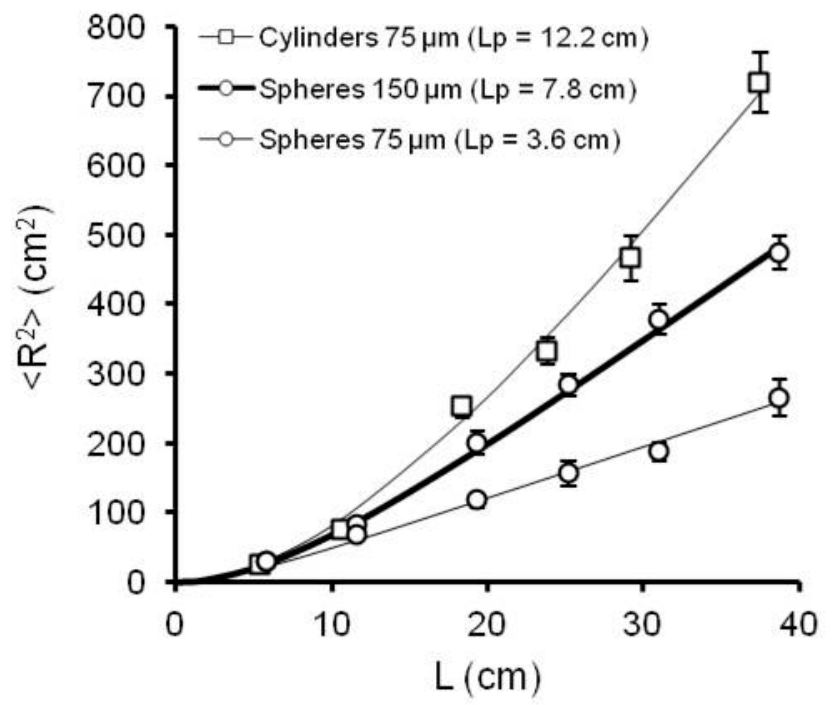

b)

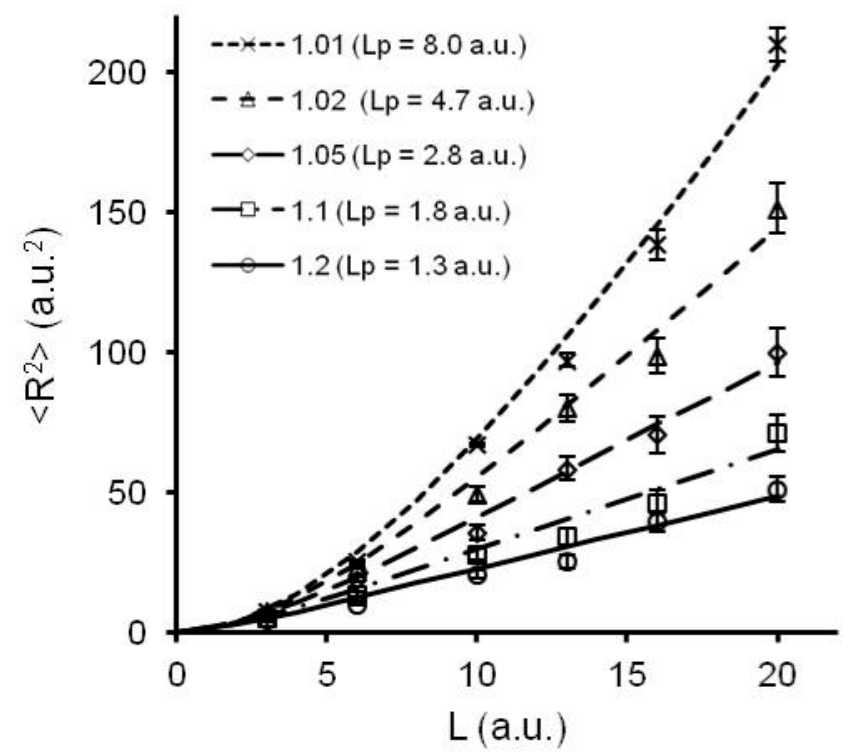


Eq. 2 predicts that the persistence length of a WLC is proportional to the bending rigidity of the polymer. The experimental results showed an increase in $L_{p}$ with the diameter of the thread; the trends described by eq. 2 are thus reproduced in the MacAg model. The WLC description was still valid when we replaced the spherical beads by cylindrical beads: $L_{p}$ increased, reflecting an increase of the rigidity of the chain. The cylinders-on-a-string is still described by the WLC model because, even though it is constructed with cylinders, the chain is still 1D, inextensible and continuously flexible; the presence of cylinders did not prevent continuous bending. The supplementary information gives the distributions of $R$ (Fig. S2).

For the WLC model, whether by simulations or analytical studies, theoretical descriptions of the distributions of $R$ are complex and still the subject of active discussion. ${ }^{25,26}$ The detailed analysis of these distributions is outside the scope of the present article. Modifying the physical properties of our simple beads-on-a-string model leads to changes in the dynamics of the system that are qualitatively compatible with the WLC analytical model. We thus infer that our macroscopic experimental system models at least some important aspects of WLC behavior, and can thus be useful in exploring conceptual aspects of the dynamics of homopolymers in solution.

\section{Monte-Carlo Simulations}

We performed Monte-Carlo simulations as described in the experimental design section.

Fig. 2b shows the evolution of $\left\langle R^{2}>\right.$ as a function of $L$ for different flexibilities of the simulated chain. We fixed the flexibility from 1.01 to 1.20 , where larger numbers correspond to more flexible chains. We fitted the experimental points with the WLC model according to eq. 1, and found good correlations $\left(r^{2}>0.98\right)$. The persistence length decreased from $L_{p}=8.0$ a.u. to $L_{p}=$ 1.3 a.u. for flexibilities going from 1.01 to 1.2 (corresponding to decreasing the stiffness of the chain). The Monte-Carlo simulation reproduced the results obtained for the macroscopic model. 
We chose parameters of the simulation to match the magnitudes of the $L / L_{p}$ ratios as the ones obtained for the experiments, and ranging from approximately 0.5 to 10 . The $L / L_{p}$ ratios reflect the flexibility of the chain: $L / L_{p}>>1$ corresponds to a flexible chain, and $L / L_{p}<<1$ corresponds to a rigid chain.

The distributions of $R$ in experiment and simulation (given in the supplementary information) are qualitatively similar for comparable $L / L_{p}$ ratios. The simulations thus provide useful insight into the parameters that are important in controlling the mechanisms of macroscopic chain dynamics. Indeed, numerous external parameters are important for this macroscopic system (e.g. friction, kinetic energy, and momentum transfer through collisions) that can be irrelevant or absent for a molecular system. Although none of these parameters is present in the Monte-Carlo simulation, we obtained the same behavior as in the macroscopic experiment. Obtaining similar dynamics for both the simulations and the macroscopic experiments confirms the universal application of the WLC model for 1D inextensible and continuously flexible objects - stiff enough to comply with the self-avoidance conditions. Both our MC model and MacAg model fulfill the necessary conditions for WLC behavior.

The macroscopic forces (e.g., gravity, friction, collision, tension) that determine the behavior of the macroscopic system are fundamentally different from the forces that regulate molecular dynamics (e.g., electronic repulsion, electrostatic and dipolar interactions, solvent interactions). Nonetheless, the WLC model apparently applies both to our macroscopic system and to stiff molecular polymers. We thus propose to use the beads-on-a-string MacAg sytem to model WLC molecules, and to include this analog system, and variants on it, in more complex designs comprising supplementary components that mimic solvents, intra-chain interactions, etc. The 2D design of the MacAg model is not a limiting characteristic in describing the dynamics of 3D systems, including molecular polymers. Indeed, using arguments we developed in the 
introduction, the MacAg model gives identical results in 2D and in 3D, as long as we consider a 1D inextensible and continuously flexible object.

\section{Effect of the Frequency of Agitation}

The macroscopic model is sensitive to the frequency of agitation $f$. Fig. 3a shows the change of $<R^{2}>$ as a function of $L$ for chains with a $150 \mu$ m-diameter thread, for three different $f$ (120, 140 and 160 rpm). As described above, we fit the data using eq. 1 and observed a decrease of the persistence length from 9.2 to $5.8 \mathrm{~cm}$ when $f$ increased from 120 to $160 \mathrm{rpm}$ (Fig. 3b). Eq. 2 indicates that $L_{p}$ for a WLC molecule is inversely proportional to the temperature, while the rigidity of the chain, $g$, does not depend on temperature. $L_{p}$ of a WLC molecule decreases with increasing $T$ and we observed a decrease of $L_{p}$ of the macroscopic chains with increasing $f$.

Temperature at the molecular scale is proportional to the average kinetic energy of the particles. ${ }^{5}$ In the MacAg model, the movement of the chain is induced by the agitation of the apparatus; as $f$ increased, the elements of the chain rolled more rapidly on the surface. The concept of "granular temperature" in granular systems has previously been defined as the fluctuation of the kinetic energy of the grains, and is related to the frequency of agitation. ${ }^{27}$

Our macroscopic system is a simple interconnected granular system, in which the beads have been linked together. Even though a detailed kinetic study is not within the scope of this paper, we did observe a significant decrease of $L_{p}$ as we increased $f$. Since we see no obvious reason for an increase of the rigidity of the chain when increasing $f$, we assume that the evolution of $L_{p}$ is related to the change in $f$. The decrease of $L_{p}$ of the macroscopic beads-on-a-string with the frequency of agitation can thus be compared to the dependence of $L_{p}$ in polymers with temperature. Further investigations are under way to deepen this understanding of the relationship between mechanical agitation of macroscopic particles and temperature at the molecular scale. 
Figure 3. a) Mean-square end-to-end distance as a function of the length of the chain for spheres threaded on a $150 \mu$ m-diameter thread with changing frequencies of agitation and their corresponding $L_{p}$ calculated using the Worm-Like Chain model. Error bars are 95\% confidence interval. b) Evolution of the fitted persistence length $L_{p}$ as a function of the frequency of agitation $f$, with standard errors.
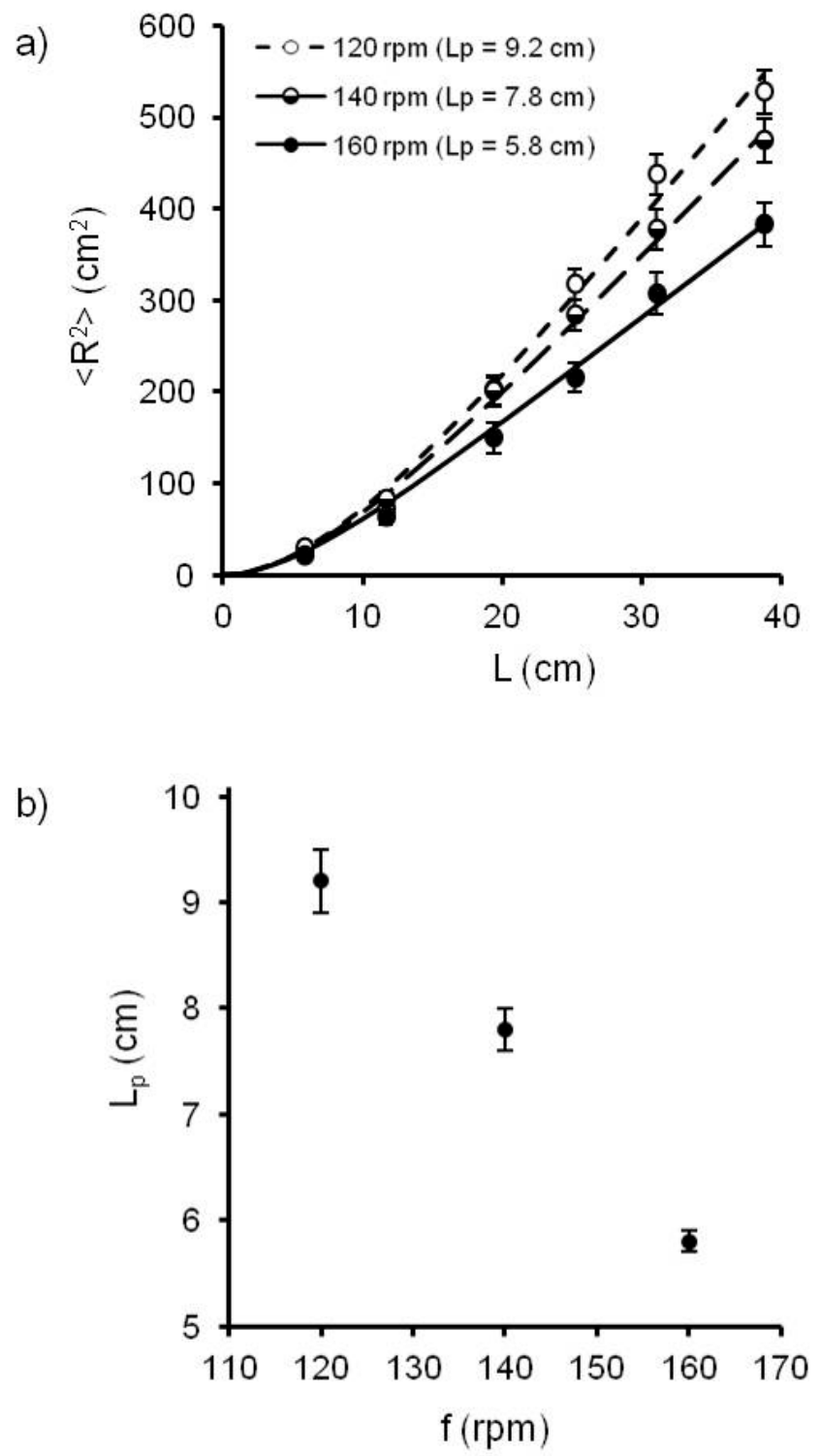


\section{Conclusion}

The macroscopic experimental system of beads-on-a-string, which we present in this paper, behaves with the same statistical description as the WLC theory - a commonly used model for stiff polymers such as DNA or short-chain unfolded proteins. The universality of the WLC description for both the 2D macroscopic MacAg model and some 3D molecular polymers is the consequence of the prerequisites of the model: $1 \mathrm{D}$, inextensible, and continuously flexible.

Nevertheless, the nature of the interactions that regulate the motion of the chains is different at the macroscopic and molecular scales, and the motion of the macroscopic beads does not follow Brownian motion. The MacAg system is thus not suitable to describe the microscopic properties of molecular motion, but it does have remarkable phenomenological similarities with statistical descriptions of molecular behavior. Its conceptual simplicity and its ease of visual characterization (due to the use of macroscopic beads), makes it an interesting complement to

analytical models and simulations. These characteristics also makes MacAg systems conceptually simple pedagogical models, and are a way to gain and test intuition.

The phenomenological similarities of our macroscopic model with WLC behavior provide new opportunities for comparing the response of the MacAg system to more complex phenomena. Simulations of phenomena such as polymer-solvent mixtures, phase transitions (e.g. coil-globule), and self-assembly are currently under investigation.

\section{Acknowledgments}

We thank Dr. Xinyu Liu for insightful discussions and assistance in data analysis. This work was supported by the US Department of Energy, Division of Materials Sciences \& Engineering, under Award No. DE-FG02-OOER45852. 


\section{References}

1. B. A. Grzybowski, A. Winkleman, J. A. Wiles, Y. Brumer and G. M. Whitesides, Nat. Mater., 2003, 2, 241-245.

2. G. K. Kaufman, M. Reches, S. W. Thomas, J. Feng, B. F. Shaw and G. M. Whitesides, Appl. Phys. Lett., 2009, 94, 044102.

3. G. K. Kaufman, S. W. Thomas, M. Reches, B. F. Shaw, J. Feng and G. M. Whitesides, Soft Matter, 2009, 5, 1188-1191.

4. M. Reches, P. W. Snyder and G. M. Whitesides, Proc. Nat. Acad. Sci., 2009, 106, 1764417649.

5. M. Plischke and B. Bergersen, Equilibrium Statistical Physics, World Scientific, 2006, p. 31 and 355.

6. P. J. Flory, Statistical Mechanics of Chain Molecules, Interscience Publishers, New-York, NY, 1969, p. 401-403.

7. B. Bammes and J. S. Olafsen, Chaos, 2004, 14, S9-S9.

8. $\quad$ E. Ben-Naim, Z. A. Daya, P. Vorobieff and R. E. Ecke, Phys. Rev. Lett., 2001, 86, 14141417.

9. $\quad$ K. Safford, Y. Kantor, M. Kardar and A. Kudrolli, Phys. Rev. E, 2009, 79, 061304.

10. L. N. Zou, X. Cheng, M. L. Rivers, H. M. Jaeger and S. R. Nagel, Science, 2009, 326, 408-410.

11. O. Kratky and G. Porod, Rec. Trav. Chim. Pays-Bas, 1949, 68, 1106-1122.

12. R. Phillips, J. Kondev and J. Theriot, Physical Biology of the Cell, Garland Science, NewYork, NY, 2009, p. 363-366.

13. J. F. Marko and E. D. Siggia, Macromolecules, 1995, 28, 8759-8770.

14. M. S. Z. Kellermayer, S. B. Smith, H. L. Granzier and C. Bustamante, Science, 1997, 276, 1112-1116.

15. J. Moukhtar, C. Faivre-Moskalenko, P. Milani, B. Audit, C. Valliant, E. Fontaine, F. Mongelard, G. Lavorel, P. St-Jean, P. Bouvet, F. Argoul and A. Arneodo, J. Phys. Chem. $B, 2010,114,5125-5143$.

16. S. Geggier, A. Kotlyar and A. Vologodskii, Nucleic Acids Res., 2010, 39, 1419-1426.

17. J. A. Schellma, Biopolymers, 1974, 13, 217-226.

18. K. A. Dill and S. Bromberg, Molecular Driving Forces, Garland Science, New-York, NY, 2011, p. 664.

19. C. Bouchiat, M. D. Wang, J. F. Allemand, T. Strick, S. M. Block and V. Croquette, Biophys. J., 1999, 76, 409-413.

20. T. E. Fisher, P. E. Marszalek and J. M. Fernandez, Nat. Struct. Biol., 2000, 7, 719-724.

21. E. Haas, M. Wilchek, E. Katchalskikatzir and I. Z. Steinberg, Proc. Nat. Acad. Sci., 1975, 72, 1807-1811.

22. M. D. Wang, H. Yin, R. Landick, J. Gelles and S. M. Block, Biophys. J., 1997, 72, 13351346.

23. S. Weiss, Science, 1999, 283, 1676-1683.

24. The details of the experimental setup are given in the materials and methods section in the supplementary information. The longest chain we studied was around $40 \mathrm{~cm}$ long (21 beads). Longer chains reached the physical limit of our apparatus, and collapsed on the side of the dish during the time of agitation.

25. H. M. Jian, A. V. Vologodskii and T. Schlick, J. Comp. Phys., 1997, 136, 168-179. 
26. S. Mehraeen, B. Sudhanshu, E. F. Koslover and A. J. Spakowitz, Phys. Rev. E, 2008, 77, 061803.

27. I. S. Aranson and L. S. Tsimring, Granular patterns, Oxford University Press, New-York, 2009, p. 42. 